\title{
Conservative versus Surgical Management of latrogenic Tracheal Rupture
}

\author{
Nikolaos Panagiotopoulos ${ }^{\mathrm{a}}$ Davide Patrini ${ }^{\mathrm{a}}$ Matthew Barnard ${ }^{\mathrm{b}}$ \\ Efstratios Koletsis $^{a, c}$ Dimitrios Dougenis ${ }^{c}$ David Lawrence ${ }^{a}$ \\ a Department of Cardiothoracic Surgery, The Heart Hospital, University College London Hospital, and \\ ${ }^{b}$ Department of Anaesthesiology and Intensive Care, St Bartholomew's Hospital, London, UK; \\ 'Department of Cardiothoracic Surgery, School of Medicine, University of Patras, Patras, Greece
}

\section{Keywords}

Tracheal rupture $\cdot$ latrogenic surgical emphysema . Intubation · Pneumomediastinum

\begin{abstract}
latrogenic tracheal rupture (ITR) represents a life-threatening condition requiring prompt diagnosis, management, and treatment. The management of ITR is challenging, and treatment options depend on tear location, size, injury extent, and the patient's respiratory status. Although this complication has been extensively reported in published literature, the best evidence practice, for the management, requires clarification. In this review, the authors focused on the establishment of a differential diagnosis and the potential mechanism of the injury, the decision-making process, and the therapeutic approaches. It is suggested that for small lacerations or stable patients, conservative management could be considered sufficient, whereas invasive surgical therapy would be more appropriate in cases of large defects with significant air leak and patient instability.
\end{abstract}

(c) 2017 S. Karger AG, Basel

\section{Introduction}

Iatrogenic tracheal rupture (ITR) represents a lifethreatening condition that requires prompt diagnosis, management, and treatment. Prokakis et al. [1] reported that prevalence of ITR in elective orotracheal intubations was $1 / 20,000-75,000$ patients and increased to $15 \%$ in emergently performed procedures, and for percutaneous dilatative tracheotomies the estimated incidence is $0.2-$ $0.7 \%$. In the last decade, the estimated incidence of ITR after endotracheal intubation is $0.05-0.37 \%$ [1], with a higher incidence of approximately $0.5-1 \%$ reported in cases of double-lumen intubation [2]. The presence of other concomitant pathologies of patients undergoing surgical procedure for this, or those who are treated in intensive care units for other reasons, could lead to an early adverse outcome, ventilator failure, airway obstruction, or death from tension pneumothorax, or late development of airway stenosis and recurrent pulmonary infections [1]. Therefore, although prompt diagnosis is essential for the survival of the patients, the diagnostic delay is $25.7 \pm 22.9 \mathrm{~h}$ (range, $3-72 \mathrm{~h}$ ) [3]. The decision making

Efstratios Koletsis, MD, MSc, PhD

Department of Cardiothoracic Surgery, University of Patras

31 Chlois Street

GR-166 73 Voula (Greece)

E-Mail ekoletsis@ hotmail.com 
regarding the treatment of these patients is highly challenging. The principal necessity is to secure and maintain the airway and to allow adequate ventilation. If a repair of the injury is to be performed, minimal impact on the respiratory function and subsequent quality of life must be ensured.

\section{Diagnosis}

In non-thoracic surgery patients, tracheal injuries were diagnosed based on presenting symptoms of dyspnoea, subcutaneous emphysema, and haemoptysis in the early postoperative period. In thoracic surgery patients, they were diagnosed intraoperatively by direct visualization. In patients intubated in intensive care units, injuries presented with subcutaneous emphysema and/or pneumothorax. Diagnosis can be established with different investigations. Chest CT could confirm the presence of pneumothorax, pneumomediastinum, mediastinitis, or pneumonia; fiberoptic bronchoscopy will allow direct evaluation of the whole trachea; oesophagoscopy may exclude an oesophageal lesion [4].

\section{Mechanisms of Injury}

ITR commonly occurs after intubation or tracheotomy procedures. The most common mechanisms are intubation by inexperienced clinicians, dilatative percutaneous tracheotomy, and change of the position of the intubation tube without deflation or overinflation of the tube cuff $[2,4]$. Relative overinflation of the tube cuff occurs if the tracheal tube is inflated just above the carina and moved back to the narrower upper portion of the trachea. Intubation with "double lumen" endotracheal tubes causes ITR more frequently, probably owing to their larger diameter and rigidity [5]. This mechanism of injury explains the incidence of trachea laceration after intraoperative repositioning of the patient's head or body, resulting in displacement of the endotracheal tube. In females, the trachea's narrower diameter, less resistant wall, and shorted length are predisposing factors to this injury [6]. Characteristically, they are longitudinal lacerations of the posterior tracheal wall $[4,6]$. These tracheal tears are frequently found on the right side because of the proximity to the oesophagus on the left side, stabilizing the trachea wall. Occasionally, tracheal lacerations can extend into the main bronchi [6].

Iatrogenic Tracheal Rupture

\section{Treatment Decision}

Deja et al. [4] described a comprehensive algorithm regarding the diagnosis and management of ITR. Conservative treatment is favoured for patients who do not require mechanical ventilation [7]. When mechanical ventilation is possible without any loss of tidal volume and the emphysema is only mild and stable during ventilation, conservative management is indicated. This choice is independent of the length of the lesion and includes lesions close to the carina $[4,6,7]$. When the injury includes the full thickness of the posterior membrane and mediastinal structures protrude into the tracheal lumen, surgical repair is indicated.

This algorithm is a more conservative approach compared to previous authors who suggest that nonsurgical therapy must only be considered in stable patients with small, uncomplicated tears of lengths between $<2$ and $<4$ $\mathrm{cm}[8,9]$. They advise that conservative treatment can be considered if previous pulmonary or mediastinal surgery has not been performed [9].

\section{Therapeutic Approaches}

In patients with spontaneous breathing at the time of diagnosis, medical treatment includes a broad-spectrum antibiotic therapy against the tracheobronchial flora, antiseptic anti-inflammatory aerosol therapy, antitussive agents, and chest tube insertion, if required [9].

In patients still requiring mechanical ventilation at the time of diagnosis due to acute respiratory failure, coma, or multi-organ failure, conservative management includes positioning the tracheal tube distal to the tracheal rupture, continuous measurement of the cuff pressure, and chest tube insertion, if required [4]. This strategy is effective in patients with lacerations in the upper region of the trachea. In cases of mechanically ventilated patients with lacerations close to the carina, which is the more common scenario, it is not easy to position the cuff distal to the tracheal rupture and to avoid dislocation of the tube tip into the mediastinum. Therefore, the tube must be sited close to the carina, under bronchoscopy with continuous cuff pressure monitoring, for a limited period [4]. In these patients, early tracheostomy allows spontaneous breathing with the aid of lower positive inspiratory pressure and continuous positive airway pressure ventilation.

Special care must be taken in patients with acute lung injury or acute respiratory distress syndrome, using pres-

Med Princ Pract 2017;26:218-220

DOI: $10.1159 / 000455859$ 
sure-controlled low tidal volume ventilation, prone positioning, and implementation of early spontaneous ventilation, inhalation of nitric oxide, and, in some patients, extracorporal membrane oxygenation or pumpless extracorporeal lung assist, postponing surgery to improve the clinical condition and gas exchange prior to the operation procedure [4].

Conservative management can be facilitated by tracheal stents, which avoid tracheal tube migration and permit early extubation [4]. Their main disadvantage is dilatation of the trachea with a potential increase in the diameter.

Welter et al. [10] reported a small series of 4 patients with rupture of the membranous wall of the trachea. They proposed complete intraluminal repair using a $70-\mathrm{cm}$, 2-0 Vicryl suture (UCLX needle; Ethicon, Germany). The suture is anchored to one end with a small absorbable polydioxanone clip (ABSOLOK AP100; Ethicon). A running suture starting at the peripheral end of the tear includes the whole posterior wall. At the proximal end of the suture, one or two polydioxanone clips are applied to secure the suture.

When surgery is indicated, it should be performed promptly to optimize success and avoid complication. The surgical approaches are dependent on which anatomical region of the tracheobronchial tree is affected [11]. For the cervical trachea, a collar incision or left cer- vicotomy for the posterior wall is indicated. A collar incision plus partial sternotomy can be performed for proximal mediastinal tracheal injuries. With a transcervicaltranstracheal approach, one can also perform a repair of the membranous wall of the trachea with subsequent closure of the tracheal incision. A right thoracotomy approach is mandatory in carina injuries, the proximal part of the left mainstem bronchus, and the right bronchial tree. Injuries of the left mainstem bronchus close to the lung hilum and left bronchial tree necessitate a left thoracotomy approach $[4,10]$.

\section{Conclusions}

The treatment decision for ITR depends on tear location, injury extent, and size. For small lacerations and stable patients, conservative management is considered sufficient, while surgical operation is mandatory in cases of large defects with significant air leak and patient instability.

\section{Disclosure Statement}

The authors declare no conflicts of interest.

\section{References}

1 Prokakis C, Koletsis E, Dedeilias P, et al: Airway trauma: a review on epidemiology, mechanisms of injury, diagnosis and treatment. J Cardiothorac Surg 2014;9:117.

2 Altinok T, Can A: Management of tracheobronchial injuries. Eurasia J Med 2014;46: 209-215.

3 Gomez-Caro Andres A, Moradiellos Diez FJ, Ausin Herrero P, et al: Successful conservative management in iatrogenic tracheobronchial injury. Ann Thorac Surg 2005;79:18721878 .

4 Deja M, Menk M, Heidenhain C, et al: Strategies for diagnosis and treatment of iatrogenic tracheal ruptures. Minerva Anestesiol 2011; 77:1155-1166.
5 Ovari A, Just T, Dommerich S, et al: Conservative management of post-intubation tracheal tears - report of three cases. J Thoracic Dis 2014;6:E85-E91.

6 Conti M, Pougeoise M, Wurtz A, et al: Management of post-intubation tracheobronchial ruptures. Chest 2006;130:412-418.

7 Schneider T, Storz K, Dienemann H, et al: Management of iatrogenic tracheobronchial injuries: a retrospective analysis of 29 cases. Ann Thorac Surg 2007;83:1960-1964.
8 Carbognani P, Bobbio A, Cattelani L, et al: Management of post-intubation membranous tracheal rupture. Ann Thorac Surg 2004; 77:406-409.

9 Jougon J, Ballester M, Choukron E, et al: Conservative treatment for post-intubation tracheobronchial rupture. Ann Thorac Surg 2000;69:216-220.

10 Welter S, Krbek Th, Hajder R, et al: A new technique for complete intraluminal repair of iatrogenic posterior tracheal lacerations. Interact Cardiovasc Thorac Surg 2011;12:6-9.

11 Koletsis E, Prokakis Ch, Baltayiannis N, et al Surgical decision making in tracheobronchial injuries on the basis of clinical evidences and the injury's anatomical setting: a retrospective analysis. Injury 2012;43:1437-1441. 